\title{
Characterization of the $\mathrm{THz}$ quantum cascade laser using fast superconducting hot electron bolometer
}

\author{
Yu. V. Lobanov ${ }^{1,2}$, Yu. B. Vakhtomin ${ }^{1,2}$, I. V. Pentin ${ }^{1,2}$, R. A. Khabibullin ${ }^{3}$, \\ N. V. Shchavruk ${ }^{3}$, K. V. Smirnov ${ }^{1,2,4}$ \\ ${ }^{1}$ LLC “Superconducting Nanotechnology” (SCONTEL), Moscow, Russia, lobanov@scontel.ru \\ ${ }^{2}$ Moscow State Pedagogical University, Moscow, Russia \\ ${ }^{3}$ V.G. Mokerov Institute of Ultra High Frequency Semiconductor Electronics of RAS, Moscow, Russia \\ ${ }^{4}$ National Research University Higher School of Economics, Moscow Institute of Electronics and Mathematics, Moscow, \\ Russia
}

Invention and demonstration of solid-state terahertz-range quantum cascade lasers (THz QCLs), followed by inevitable development of the fabrication technology, brought in a new era for the terahertzrange related fundamental and practical applications, such as spectroscopy and astrophysics, imaging and communication. With increase of the THz QCL research papers (with details found elsewhere, e.g., [14]), optimization of the lasing characteristics, improvement of its performance and development of the laser characterization techniques attracts a special attention among the researchers. There is a wide variety of different techniques for the detection of $\mathrm{THz}$ QCL. However, most of them are based on incoherent thermal detection with the slow time-constant of thermal process $\sim 1 \mathrm{~ms}$. In this paper, we present a new characterization method of THz QCL operated in the pulsed mode. In the proposed method the $\mathrm{THz}$ QCL is biased by applying a smooth gradual (i.e., with a "slow-rising" front edge) voltage pulse, and the laser output power was measured using $\mathrm{NbN}$ superconducting hot electron bolometer (HEB) within a single voltage pulse front edge, which was possible due to sufficiently short response time (of the order of $1 \mathrm{~ns}$ ) of the HEB. The HEB which we used was similar to that described elsewhere [5]. The primary advantage of this method over the most common $\mathrm{THz}$ detection techniques derives from its ability to measure THz QCL output power-current relationship within a single voltage pulse. In addition, the use of a smooth gradual voltage pulse makes it possible to avoid strong fluctuations of the current flowing through the THz QCL at the beginning and the end of the driving pulse in the case of using a driving pulse with sharp edges [6]. In our setup, THz QCL was mounted onto a cold plate of a closed-cycle refrigerator, whose base temperature was varied in a range of $5-60 \mathrm{~K}$. Principally, a very short measurement time set by duration of the driving pulse and its repetition rate $(\sim 5 \mu \mathrm{s}$ and $200 \mathrm{~Hz}$, respectively), prevents the QCL from overheating by the driving current. Amplitude of the driving pulse was chosen to be slightly higher than the laser threshold voltage. Fig. 1 depicts a driving pulse along with the measured lasing power which is given in arbitrary units. THz radiation was focused by a couple of off-axis parabolic mirrors onto the detector block. HEB was installed onto a cold plate of a wet helium cryostat. DC voltage and a resistive heater were used to bias the HEB to its optimal bias point where a high responsivity is obtained.
$\mathrm{THz}$ QCL power incident on the detector was kept within detector's dynamic range. The HEB signal was amplified using a low noise cold amplifier and an $\mathrm{R} \& \mathrm{~S}$ oscilloscope was used for the signal analysis.

$\mathrm{THz}$ QCL based on four tunnel-coupled quantum wells $\mathrm{GaAs} / \mathrm{Al}_{0.15} \mathrm{Ga}_{0.85} \mathrm{As}$ with a resonance-phonon depopulation scheme emitting near $2.3 \mathrm{THz}$ was investigated by the proposed method. The laser was processed into a double metal waveguide based on $\mathrm{Au}$ with dimensions $1 \mathrm{~mm} \times 100 \mu \mathrm{m} \times 10 \mu \mathrm{m}$ using the conventional technology described in [7]. The laser is die bonded to a copper submount with wire bonding to ridge structures. No coatings or lens were deposited on the laser facet. A THz QCL emits radiation when the value of voltage exceeds the laser threshold level (i.e. when the laser gain coefficient is exactly balanced by the sum of waveguide and mirror losses). Applying a smooth gradual voltage pulse it becomes possible to smoothly approach the threshold condition which was examined at different operating temperatures of the QCL. As deduced from data given in Fig. 1, the laser threshold and peak power are achieved at voltages of $10.7 \mathrm{~V}$ and $10.8 \mathrm{~V}$ at $5 \mathrm{~K}$, respectively. A further increase in the laser voltage is accompanied by a sharp drop in the optical power until lasing ceases at $11.3 \mathrm{~V}$. After a sharp increase in output power beyond the laser threshold,

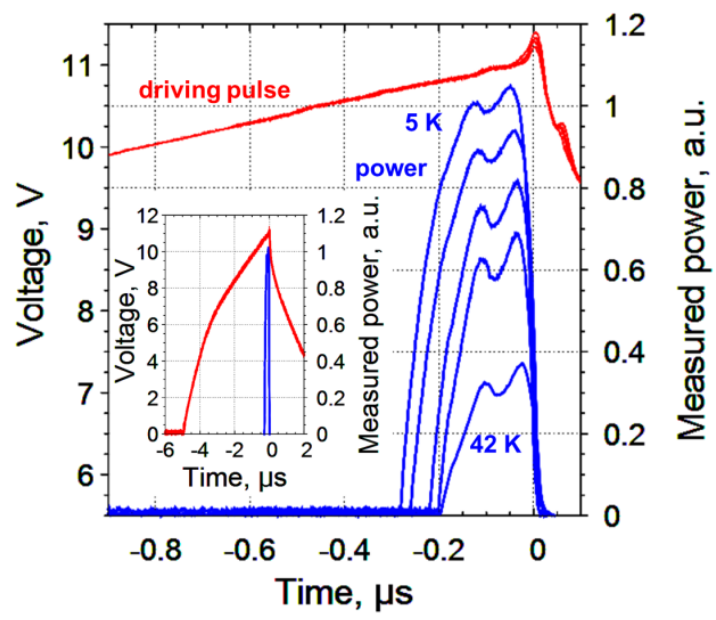

Fig. 1. QCL driving voltage pulse and measured lasing power registered with HEB. With increase of the QCL operating temperature from 5 to $60 \mathrm{~K}$ lasing power has decreased. In the figure, measured power at $5,14,36,41$, and $42 \mathrm{~K}$ is shown. The lasing pulse width has also decreased from $\sim 250 \mathrm{~ns}$ at $5 \mathrm{~K}$ down to $\sim 100 \mathrm{~ns}$ at $56 \mathrm{~K}$. Inset shows rise and decay of the voltage pulse and relative position of the emitted $\mathrm{THz}$ pulse during the driving pulse 
a nonmonotonic behavior of the output power-current dependence is observed. We suppose that this is due to the mode hopping effect with an increase of applied electric field. Commonly, the gain maximum shifts to higher frequencies with an increase in applied electric field, which is related to transformation of the laser levels positions [8]. The influence of temperature on the laser parameters is one of the most important characteristic of THz QCL. The temperature dependence of the threshold current $J_{t h}$ was measured by the proposed method. Using a phenomenological relationship for the temperature dependence:

$$
J_{t h}=J_{1}+e^{T / T_{0}},
$$

we determined the characteristic temperature $T_{0}$ to be $\sim 26 \mathrm{~K}$. Since in our experiment we have measured rather voltage, the current was obtained using a mathematically derived fitting parameter $A R$ such that $J_{t h}=V_{t h} / A R$, with $A R=0.0217 . J_{t h}(T)$ and its fit according to (1) are depicted in Fig. 2. Following a paper by Albo \& Hu [9] we have also examined $P(T)$ dependence of the QCL power and experimental results are shown in Fig. 3. The presence of second plateaus in $P(T)$ in the temperature range from 43 to $52 \mathrm{~K}$ is not traditional for this characteristic.

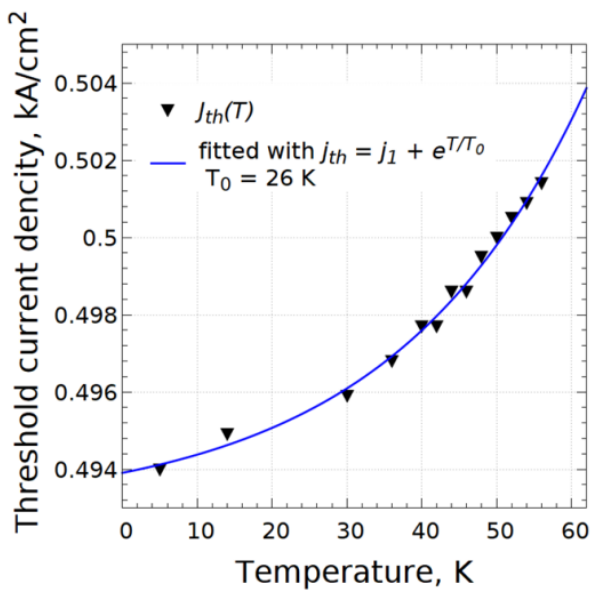

Fig. 2. Threshold current dependence on the QCL operating temperature. Experimental points are fitted according to (1)

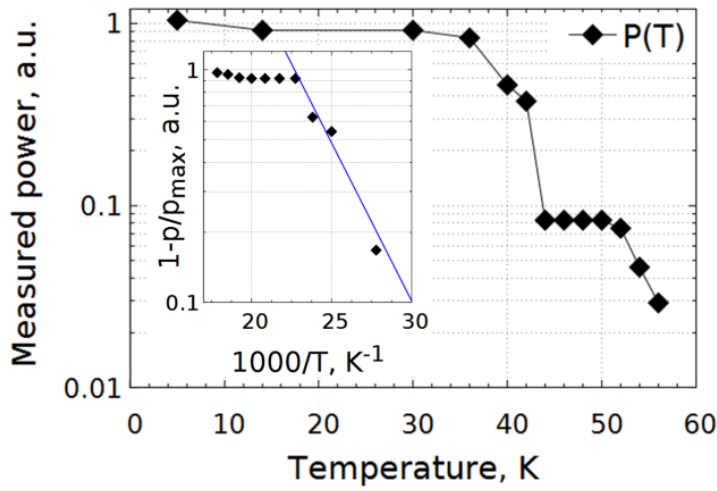

Fig. 3. Dependence of the laser output power (normalized) on the QCL operating temperature. Inset shows the Arrhenius plot with a fitting line in the temperature range 30$42 \mathrm{~K}$, from which an activation energy $E_{a}$ of $\sim 26 \mathrm{meV}$ has been derived using (2)
Typically $P(T)$ gradually decreases with increasing temperature and at high temperatures the dominant mechanism of scattering is thermally activated LO-phonon scattering [9]. The formation of the second plateau in $P(T)$ is most likely caused by the leakage of electrons from lower laser level to the continuum. Thus, a partial compensation of "negative" thermally activated LO-phonon scattering and "positive" leakage of electrons from lower laser level is obtained. Nevertheless, Arrhenius plot of the $P(T)$ dependence (inset in Fig. 3) is well fitted to:

$$
\frac{P_{\text {out }}(T)}{P_{\text {out }}^{\max }}=1-\alpha e^{-E_{a} / k T}
$$

in the $30-42 \mathrm{~K}$ temperature range, providing an activation energy $E_{a}$ of $\sim 26 \mathrm{meV}$ which is reasonably close to a calculated value of $\sim 28 \mathrm{meV}$.

In conclusion, we have proposed and demonstrated a new approach for THz-QCL characterization allowing $P\left(V_{\text {bias }}\right), P(T)$, and $I_{t h}(T)$ analysis. Obtained results are in a reasonable agreement with previous results; however, further study of these dependencies may reveal additional information regarding the QCL structure. Exploiting fast response of our detector and tunability of the implemented pulsed gradual biasing a thorough investigation of the QCL emittance depending on the driving parameters could be carried out.

This research has been partially supported by the RSF grant No. 17-72-30036 (development and implementation of the experimental technique, data analysis) and RFBR grant No. 17-02-00070 A (design and processing of the QCL chip, data analysis).

\section{References}

1. Williams, B. S. Terahertz quantum-cascade lasers // Nat. Photonics 2007. V. 1. P. 517-525.

2. Scalari, G., et al., THz and sub-THz quantum cascade lasers // Laser \& Photon. Rev. 2009. V. 3, No. 1-2. P. 45-66.

3. Sirtori, C., et al., Wave engineering with THz quantum cascade lasers Nat. Photonics 2013. V. 7. P. 691-701.

4. Liang, G., et al., Recent Developments of Terahertz Quantum Cascade Lasers // IEEE J. Sel. Top. Quant. 2017. V. 23, Iss. 4. P 1-18.

5. Seliverstov, S., et al., Fast and sensitive terahertz direct detector based on superconducting antenna-coupled hot electron bolometer // IEEE Trans. on Appl. Supercond. 2015. V. 25, No. 3. P. 2300304-1 - 23003045.

6. Scheuring, A., et al., Transient Analysis of THzQCL Pulses Using NbN and YBCO Superconducting Detectors // IEEE Trans. THz Sci. Techn. 2013. V.3, No. 2. P. 172-179.

7. Khabibullin, R. A., et al., Fabrication of a terahertz quantum-cascade laser with a double metal waveguide based on multilayer GaAs/AlGaAs heterostructures // Semiconductors. 2016. V. 50, No. 10. P. 1377-1382.

8. Khabibullin, R. A., et al., Temperature dependences of the threshold current and output power of a quantumcascade laser emitting at $3.3 \mathrm{THz} / /$ Semiconductors. 2018. V. 52, No. 11. P. 1381-1386.

9. Albo, $A$., $H u, Q$. Investigating temperature degradation in THz quantum cascade lasers by examination of temperature dependence of output power // Appl. Phys. Lett. 2015. V. 106. P. 131108-1 - 131108-5. 